\title{
Outcomes of young patients diagnosed with locally advanced rectal cancer
}

\author{
Rosemary Habib ${ }^{1,2,3,4}$, Nicholas G. Burgess ${ }^{3,5}$, Michael J. Bourke ${ }^{3,5}$, Mark Wong ${ }^{2,3,6}$, Nicholas \\ Wilcken $^{2,3}$, James Toh ${ }^{3,7}$, Toufic El-Khoury, ${ }^{3,7}$, Nimalan Pathma-Nathan ${ }^{7}$, Grahame Ctercteko ${ }^{7}$, \\ Jayasingham Jayamohan ${ }^{3,9}$, Kenneth Micklethwaite ${ }^{1,3,4,10}$, Adnan Nagrial ${ }^{2,3,6}$
}

${ }^{1}$ Westmead Clinical School, The University of Sydney, Sydney, Australia; ${ }^{2}$ Department of Medical Oncology, Westmead Hospital, Crown Princess Mary Cancer Centre, Sydney, New South Wales, Australia; ${ }^{3}$ Westmead Clinical School, The University of Sydney, Sydney, New South Wales, Australia; ${ }^{4}$ The Westmead Institute for Medical Research, Westmead, New South Wales, Australia; ${ }^{5}$ Department of Gastroenterology and Hepatology, Westmead Hospital, Sydney, New South Wales, Australia; ${ }^{6}$ Blacktown hospital, Blacktown Cancer and Haematology Centre, Sydney, New South Wales, Australia; ${ }^{7}$ Division of Colorectal Surgery, Department of Surgery, Westmead Hospital, Sydney, New South Wales, Australia; ${ }^{8}$ University of Notre Dame, Sydney, New South Wales, Australia; ${ }^{9}$ Department of Radiation Oncology, Westmead Hospital, Crown Princess Mary Cancer Centre, Sydney, New South Wales, Australia; ${ }^{10}$ Sydney Cellular Therapies Laboratory, Blood and Bone Marrow Transplant Unit, Department of Haematology, Westmead Hospital, Sydney, NSW, Australia

Contributions: (I) Conception and design: A Nagrial; (II) Administrative support: A Nagrial, R Habib; (III) Provision of study materials or patients: A Nagrial, NG Burgess, MJ Bourke; (IV) Collection and assembly of data: R Habib; (V) Data analysis and interpretation: R Habib, A Nagrial; (VI) Manuscript writing: All authors; (VII) Final approval of manuscript: All authors.

Correspondence to: Adnan Nagrial, FRACP, PhD. Crown Princess Mary Cancer Centre, Westmead Hospital, Westmead Hospital Level 1, Cnr Hawkesbury and Darcy Roads, Westmead NSW 2148, Australia. Email: Adnan.nagrial@sydney.edu.au.

Background: The incidence of rectal cancer is higher in the older population. In developed nations, there has been a rise in incidence in young onset colorectal cancer (CRC). We examined the outcomes of locally advanced rectal cancer (LARC) in younger patients (yRC) compared with older patients, using a retrospective audit.

Methods: All cases of LARC referred to two tertiary referral cancer centres in Western Sydney were examined. Patient demographics, presenting symptoms, treatment, relapse free survival (RFS), overall survival (OS) and progression free survival (PFS) were obtained. Under 50 years old was used as the cut-off age for defining yRC.

Results: All 145 consecutive patients were treated for LARC, including 28 in the yRC and 117 in the older patient group. Median follow-up was 54 months. yRC were more likely to complete neoadjuvant therapy $(100 \%$ vs. $86 \% ; \mathrm{P}=0.032)$ and to undergo more extensive surgical procedures $(24 \%$ vs. $2 \%, \mathrm{P}<0.0001)$. yRC were more likely to have microsatellite high (MSI) tumours ( $30 \%$ vs. 4.7\%; $\mathrm{P}=0.003)$. yRC demonstrated significantly poorer RFS compared with the standard group (HR 2.79; median RFS 4.67 vs. 16.02 months; $\mathrm{P}=0.023$ ). In the relapsed setting, yRC had poorer PFS compared with the standard group (median PFS 2.66 vs. 9.70, $\mathrm{P}=0.006, \mathrm{HR}$ 3.04). A difference in OS was also seen between the two groups, with yRC demonstrating poorer OS (median OS 40.46 vs. 58.26 months, HR 3.48, $\mathrm{P}=0.036$ ).

Conclusions: Patients under 50 years with LARC are more likely to have MSI tumours with a more aggressive disease course and poorer RFS, PFS and OS. Initiatives to improve early detection of these patients may improve outcomes. Further research is necessary to understand this disease and optimise its treatment.

Keywords: Rectal cancer; colorectal cancer (CRC); rectal bleeding; cancer; neoadjuvant treatment

Submitted Jul 27, 2020. Accepted for publication Nov 03, 2020.

doi: 10.21037/jgo-20-300

View this article at: http://dx.doi.org/10.21037/jgo-20-300 


\section{Introduction}

The incidence of colorectal cancer (CRC) remains high in Western countries despite the overall decline in incidence worldwide (1-3). It is the third most diagnosed cancer worldwide and the second leading cause of cancer-related deaths after lung cancer (4). According to the world health organisation (WHO), in 2018, the age-standardised incidence of CRC was highest in developed countries most notably Australia and New Zealand, followed by Europe and North America (5).

The last two decades have seen significant improvements in CRC early detection, therapies, and survival, owing to a rise in translational and clinical research in the field (6-11). Despite this, locally advanced and metastatic disease are still associated with high mortality, with a 5 -year mortality rate of $30 \%$ for stage III CRC and $86.6 \%$ for stage IV disease. The majority of patients with stage IV CRC succumb to the disease over a median of 24 months $(12,13)$.

Whilst the incidence of CRC is skewed towards an older patient population, a rise in incidence has been observed in patients younger than 50 years of age $(14,15)$. One third of locally advanced rectal cancer (LARC) patients are younger than $50(16,17)$. The prognosis of young onset rectal cancer (yRC) as a subgroup, however, is uncertain as results of retrospective audits to date have either been conflicting (18-20) or have not focussed specifically on rectal cancer (20-22). Another limiting factor is the use of different age limits for defining yRC (23), and often varies in the literature to cut-offs between 35 and 50 years of age. Whilst it is logical to assume a cut-off age of $<50$ given the fact that most screening programs begin from the age of 50, clear definitions of young onset CRC and very early onset CRC are yet to be formally established (24).

Against this background, our study examines the treatment-related outcomes of LARC (including relapse and mortality) in young versus older patients who receive neoadjuvant radiotherapy. We present the following study in accordance with the STROBE reporting checklist (available at http://dx.doi.org/10.21037/jgo-20-300).

\section{Methods}

\section{Patient selection}

We conducted a retrospective review of consecutive patients with LARC (defined as Tumour stage of 3 or 4 or Nodal stage of 1 plus any tumour stage) who received neoadjuvant radiotherapy in two tertiary institutions of the Western
Sydney Local Health district (WSLHD), Sydney between January 1, 2009 and December 31, 2016. In this study, patients were separated into two groups according to their age at inclusion; the standard group was defined by the age cut-off of $\geq 50$ years old and a yRC group ( $<50$ years old). Patients were included if they had biopsy proven rectal adenocarcinoma and who were eligible for neoadjuvant chemoradiotherapy as determined by their pre-treatment staging on MRI or rigid endoscopy. Exclusion criteria included a histological diagnosis other than adenocarcinoma or mucinous adenocarcinoma, a prior history of CRC diagnosis, incomplete follow-up and if systemic treatment was delivered at other institutions. Patients who presented with de novo stage IV rectal cancer and those with an ECOG performance status of IV were also excluded from the analysis. Data were collected retrospectively and analysed anonymously. The study was performed in accordance with the standards of the Declaration of Helsinki (as revised in 2013) and was approved by the institutional ethics board of the Western Sydney Local Health District ethics committee (IBR: 1811-15 QA). No informed consent was required as this was a retrospective audit.

\section{Measures of outcome}

The diagnosis of LARC required a combination of tissue biopsy and imaging. All diagnoses were biopsy proven. The extent of disease was confirmed using computed tomography (CT) or magnetic resonance imaging (MRI). All diagnoses were biopsy proven rectal carcinomas including adenocarcinomas, mucinous or signet ring carcinomas. Squamous cell pathologies and sarcomas were excluded from the analysis.

Data was extracted for relapse free survival (RFS), progression free survival (PFS) and overall survival (OS). RFS was defined as the time from initial biopsy proven rectal carcinoma to the first event of local recurrence or metastatic recurrence. PFS was defined as the time from the diagnosis of a first recurrence to the time of progression on first line palliative chemotherapy or death from rectal carcinoma or other causes or censored to the last follow-up time. OS was defined as the time from initial biopsy proven rectal carcinoma to the time of death from rectal carcinoma or other causes or censored to the last follow-up time. RFS and OS were calculated for all patients in the study. PFS was calculated for patients who experienced disease progression.

Other data included in the analysis were patient demographics (age, sex, and comorbidities), clinical 
presentation of symptoms, tumour stage at diagnosis, doses of neoadjuvant radiotherapy and duration and whether adjuvant treatment was offered subsequent to their surgery. Completion of neoadjuvant radiotherapy was considered if patients received the full dose of standard radiotherapy for either a planned short or long course treatment. Patients undergoing neoadjuvant long course radiation received 25 daily fractions of RT with or without radio-sensitising 5 fluorouracil (5-FU) based chemotherapy to a total dose of 45 Gy. An optional boost of 5.4 Gy in 3 fractions to a total of 50.4 Gy in 28 days was given as determined by the treating radiation oncologist. Alternatively, patients received a short course of radiotherapy with 5 daily fractions to a total of 25 Gy.

Data analysis included types of surgical procedures. Surgical margins were assessed as involved as follows: for upper rectal tumours, a positive margin was defined as a distal margin of transection of $<5 \mathrm{~cm}$; and for mid rectal tumours a distal margin of $<1 \mathrm{~cm}(25)$. Rates of postoperative complications were also analysed, including infections, anastomotic leak, deconditioning leading to delay in initiation of adjuvant chemotherapy and death within 30 days post-operatively.

Histological features including postoperative stage (according to the AJCC classification, including tumour, node, metastasis), tumour grade and microsatellite instability were analysed. Pathological complete response (pCR) was defined as the absence of residual tumour cells on histopathologic examination of the rectal primary and resected lymph nodes. Other features including sites of recurrence, number of metastases and the types of palliative treatments (surgical, chemotherapy or radiotherapy) were also assessed.

\section{Statistical analyses}

Comparisons of the baseline characteristics between the two groups were performed using the $\chi^{2}$ test for categorical variables and $t$-test for continuous variables. RFS, PS and OS curves were generated using the Kaplan-Meier method, and differences in the survival rates between the groups were compared by the log-rank test. The Cox proportional hazard model was used to estimate hazard ratios (HRs) and $95 \%$ confidence intervals $(95 \% \mathrm{CI})$ of potential prognostic factors for RFS and OS.

Factors included in the univariate analyses for OS were the following: age, sex, Eastern Cooperative Oncology Group (ECOG) performance status (PS), pre-operative nodal stage, neoadjuvant therapy, histopathology, Lymphovascular invasion (LVI) and perineural invasion (PNI), immunohistochemistry for mismatch repair (MMR), post-operative nodal stage and adjuvant chemotherapy. For the relapsed patients, liver metastases and surgical treatment of metastases were additionally included in univariate analyses. Those cases with missing data relevant to the statistical analysis were excluded from the analysis. Variables with a $\mathrm{P}$ value $\leq 0.5$ in the univariate analysis were subsequently included in the multivariate analysis. The cut-off value of continuous variables was determined based on the median value. A two-sided $\mathrm{P}<0.05$ was considered statistically significant. All statistical analyses were performed using IBM SPSS ${ }^{\circledR}$ software platform version 26.

\section{Results}

\section{Patient characteristics}

Baseline patient characteristics are listed in Table 1. From January 12009 to December 31 2016, a total of 212 patients presented to our institution with a diagnosis of LARC or de novo stage IV rectal cancer. Twenty-three patients were excluded based on the aforementioned exclusion criteria. Of the 189 patients, 44 (23\%) patients had de novo stage IV disease and were subsequently excluded from the analysis, while145 (77\%) patients were treated for LARC. The median age of the study population was 62 (range, 27-91). Median follow-up was 54 months at data cut-off.

The locally advanced cohort comprised 28 (19.3\%) yRC patients and 117 (80.7\%) were in the standard group (Table 1). The median age in yRC group was 42 (range, 27-49), and 64 (range, 50-91 years), in the standard group.

$\mathrm{yRC}$ had an even proportion of males and females, whereas the older cohort had a predominantly male population, however this was not statistically significant. Most patients had an ECOG performance status of $<2$, however the older group were more likely to have a poorer ECOG status $(\geq 2)$. There were no differences in symptoms at presentation with per rectal bleeding being the most common presenting symptom in both groups, followed by altered bowel habits (constipation or diarrhoea) and anaemia.

No differences were seen in the preoperative staging. Pre-operative MRI and PET scans were used at similar rates in both groups as additional means of assessing for local and distant metastatic disease. $\mathrm{yRC}$ were more likely to complete neoadjuvant radiotherapy $(100 \%$ vs. $86 \%$, 
Table 1 Baseline demographics, pre-operative, and surgical data for the standard and yRC group

\begin{tabular}{|c|c|c|c|}
\hline Variable & yRC $(n=28)$ & Standard group $(n=117)$ & $P$ value \\
\hline Gender (male/female) & $14(50 \%) / 14(50 \%)$ & $70(60 \%) / 47(40 \%)$ & 0.344 \\
\hline ECOG Performance status & & & 0.018 \\
\hline 0 & $25(89 \%)$ & $67(57.3 \%)$ & \\
\hline II & $0(0 \%)$ & $6(5.1 \%)$ & \\
\hline III & $0(0 \%)$ & $5(4.3 \%)$ & \\
\hline Missing & $0(0 \%)$ & $1(0.85 \%)$ & \\
\hline \multicolumn{4}{|l|}{ Symptoms at diagnosis } \\
\hline \multicolumn{4}{|l|}{ Pre-operative staging } \\
\hline MRI & $24(86 \%)$ & $91(80 \%)$ & 0.527 \\
\hline PET & 27 (96\%) & $101(89 \%)$ & 0.248 \\
\hline Pre-operative stage & & & 0.667 \\
\hline 1 & $0(0 \%)$ & $2(2 \%)$ & \\
\hline 2 & $10(36 \%)$ & $35(30 \%)$ & \\
\hline 3 & $18(64 \%)$ & $62(53 \%)$ & \\
\hline \multicolumn{4}{|l|}{ Surgical treatment } \\
\hline No surgical treatment & $3(11 \%)$ & $24(21 \%)$ & 0.232 \\
\hline Surgical treatment & & & $<0.0001$ \\
\hline APR & $4(16 \%)$ & $24(25.8 \%)$ & \\
\hline LAR & $6(24 \%)$ & $33(35.4 \%)$ & \\
\hline ULAR & $8(32 \%)$ & $32(34.4 \%)$ & \\
\hline Colectomy/exenteration & $6(24 \%)$ & $2(2.2 \%)$ & \\
\hline Other & $1(4 \%)$ & $2(2.2 \%)$ & \\
\hline Postoperative complications & $5(20 \%)$ & $14(15 \%)$ & 0.509 \\
\hline
\end{tabular}

$\mathrm{P}=0.032)$. yRC patients were more likely to receive long course neoadjuvant radiotherapy $(\mathrm{P}=0.040)$. Additionally, the average time to starting radiotherapy from diagnosis for yRC was shorter than that for the standard group (median
3.8 vs. 4.7 weeks; $\mathrm{P}=0.002)$. However, no differences were observed for time to surgery from completion of neoadjuvant therapy in both groups (median of 7.5 weeks for both, $\mathrm{P}=0.451$ ). 


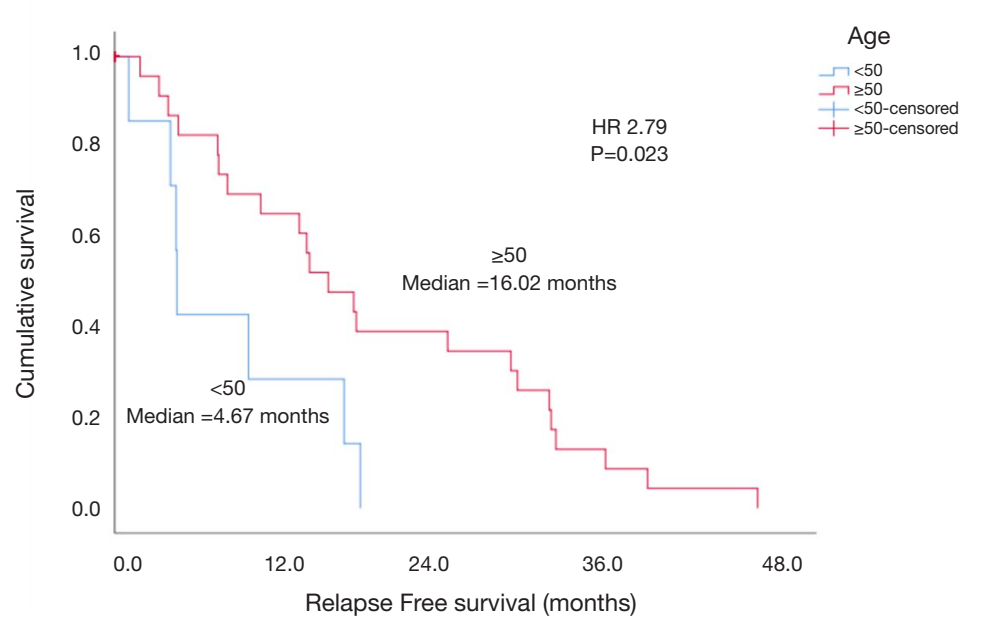

Figure 1 RFS by age. Red: standard group, blue: yRC ( $\mathrm{P}=0.023)$. RFS, relapse free survival; yRC, younger patients.

Following completion of neoadjuvant radiotherapy, a combined total of $118(81 \%)$ in both groups underwent surgical resection of the primary rectal cancer, including abdominal perineal resection in $24 \%$, low anterior resection in $33 \%$ and ultralow anterior resection in $34 \%$. yRC however, were more likely to undergo extensive surgical procedures, including total colectomies and exenterations ( $24 \%$ vs. $2 \% ; \mathrm{P}<0.0001)$. There were no differences in the rates of postoperative complications between the two cohorts.

Postoperatively, both groups had comparable histopathology results including overall stage, histology, lymphovascular and perineural invasion. Both achieved the same rates of pCR (8\%). However, yRC were more likely to have microsatellite unstable tumours $(30 \% \mathrm{vs}$. $4.7 \% ; \mathrm{P}=0.003$ ). Both groups had similar rates of adjuvant chemotherapy use (Table S1).

\section{Survival outcomes}

\section{yRC had poorer RFS post neoadjuvant radiotherapy} and surgery

Overall, 30 patients relapsed with a median time to relapse of 14 months (range, 1.05-48.22 months). Rates (28\% for yRC and $31 \%$ in the standard group; Table S1) and patterns of relapse were similar between the two groups. yRC had significantly poorer RFS compared with the standard group (HR 2.79; median RFS 4.67 vs. 16.02 months; $\mathrm{P}=0.023$ ) (Figure 1).

Results of the univariate and multivariate analyses relating to RFS are reported in Table 2. In the univariate analysis, factors associated with poorer RFS, other than age, included male gender (HR 2.592, median RFS 10.03 vs. 18.13 months; $\mathrm{P}=0.021$ ) (Table 2) as well as lack of chemotherapy use in the adjuvant setting (HR 4.087; median RFS 4.77 vs. 18.42 months; $\mathrm{P}=0.001$ ), whereas postoperative tumour grade, nodal stage and overall stage did not demonstrate a significant effect on RFS on univariate analysis (Table 2). Multivariate analysis results are reported in Table 2. Male gender and adjuvant chemotherapy use maintained their independent effect on RFS. Age did not remain an independent factor.

\section{Age was not a significant prognostic factor for OS}

Age was not a significant prognostic factor for OS (median 93.6 months vs. not reached for $\mathrm{yRC}, \mathrm{P}=0.235$ ) (Figure 2).

The factors that were significant for poorer OS on univariate analysis were perineural invasion $(\mathrm{P}=0.003$, HR 3.99), and lack of adjuvant chemotherapy $(\mathrm{P}=0.001)$ (Table 3). Post-operative $\mathrm{N}$-stage and lack of adjuvant chemotherapy use significantly correlated with survival on multivariate analysis (Table 3).

\section{Relapsed disease}

yRC with relapsed disease had poorer progression free and OS compared with the standard group

Of the 30 patients who relapsed, 23 patients received palliative chemotherapy, including $7 \mathrm{yRC}$ and 16 in the standard group. There were no statistically significant 
Table 2 Univariate and multivariate Cox proportional hazards for relapse free survival

\begin{tabular}{lll}
\hline Variable & Univariate analysis & Multivariate analysis \\
\hline Age (ref: yRC) & $\mathrm{P}=0.029, \mathrm{HR} 2.791,95 \% \mathrm{Cl}: 1.113-6.999$ & $\mathrm{P}=0.062, \mathrm{HR} 0.076,95 \% \mathrm{Cl}: 0.005-1.139$ \\
Gender (ref: male) & $\mathrm{P}=0.021, \mathrm{HR} 2.592,95 \% \mathrm{Cl}: 1.152-5.830$ & $\mathrm{P}=0.05, \mathrm{HR}$ 19.5, 95\% Cl: 2.43-156.34 \\
Lack of adjuvant chemotherapy & $\mathrm{P}=0.001, \mathrm{HR} \mathrm{4.087,95 \%} \mathrm{Cl:} \mathrm{1.723-9.693}$ & $\mathrm{P}=0.021, \mathrm{HR} 14.065,95 \% \mathrm{Cl}: 1.495-132.33$ \\
ECOG & $\mathrm{P}=0.422$ & $\mathrm{P}=0.362$ \\
Histopathology (ref: adenocarcinoma) & $\mathrm{P}=0.141$ & $\mathrm{P}=0.072$ \\
LVI & $\mathrm{P}=0.123$ & $\mathrm{P}=0.870$ \\
$\mathrm{PNI}$ & $\mathrm{P}=0.146$ & $\mathrm{P}=0.138$ \\
MMR & $\mathrm{P}=0.118$ & $\mathrm{P}=0.941$ \\
\hline
\end{tabular}

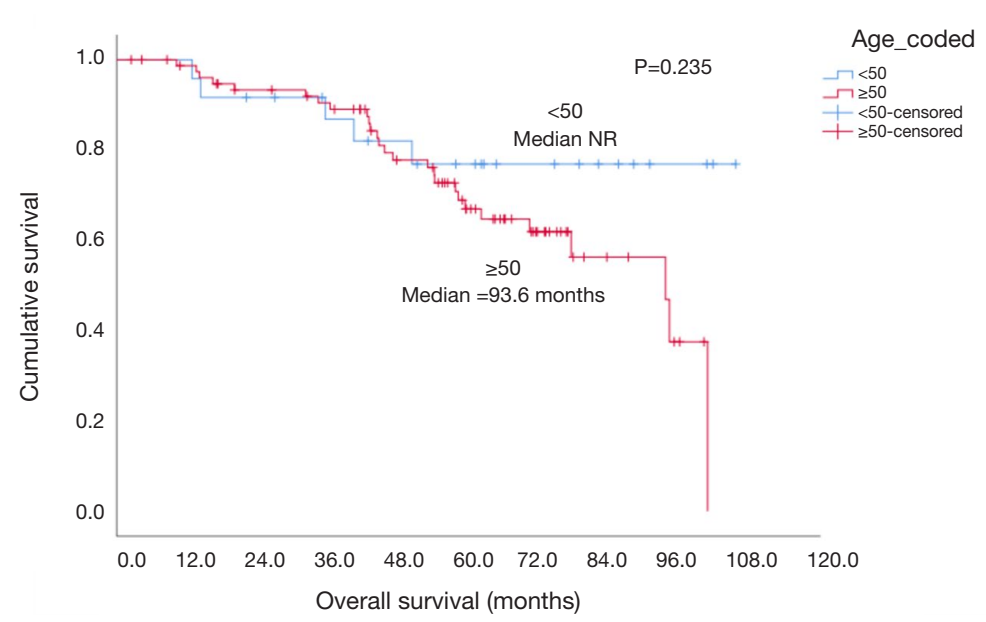

Figure 2 OS for young onset rectal cancer compared with the standard group $(\mathrm{P}=0.235)$. OS, overall survival.

differences in the number or sites of recurrence of relapsed disease in $\mathrm{yRC}$ compared with the standard cohort.

A statistically significant difference in the median PFS following palliative chemotherapy was seen, with yRC demonstrating poorer PFS compared with the standard group (median PFS 2.66 vs. 9.70, $\mathrm{P}=0.006, \mathrm{HR} 3.04$ ). 26 patients (87\%) died following relapse (7/7 in yRC and 19/23 in the standard group, $\mathrm{P}=0.236$ ). A difference in $\mathrm{OS}$ was also seen between the two groups, with yRC demonstrating poorer OS (median OS 40.46 vs. 58.26 months, HR 3.48, $\mathrm{P}=0.036$ ) (Figure 3).

Factors associated with OS are summarised in Table 4. Surgical resection of metastases improved OS (HR 4.18, median OS 94.2 vs. 50.36 months, $\mathrm{P}=0.016$ ) (Table 4). Age, mucinous histology, and surgical treatment of metastases remained significant on multivariate analysis (Table 4).

\section{Discussion}

Our study demonstrates that $\mathrm{yRC}$ with LARC have poorer survival outcomes compared with older patients despite having a better ECOG performance status, completing neoadjuvant radiotherapy at higher rates, and subsequently undergoing more extensive surgical resections. yRC demonstrated shorter RFS, and in the relapsed setting experienced shorter PFS and resultant shorter OS. yRC was also more likely to have microsatellite unstable tumours. Taken together, these data suggest a more aggressive tumour biology and potentially more resistance to palliative treatments. 
Table 3 Univariate and multivariate analyses for overall survival

\begin{tabular}{|c|c|c|}
\hline Variable & Univariate analysis & Multivariate analysis \\
\hline Gender (ref: male) & $P=0.606$ & $P=0.354$ \\
\hline Post-operative $\mathrm{N}$ stage & $\mathrm{P}=0.020, \mathrm{HR} 1.53,95 \% \mathrm{Cl}: 1.07-2.18$ & $\mathrm{P}=0.001, \mathrm{HR} 2.85,95 \% \mathrm{Cl}: 1.56-5.21$ \\
\hline Histopathology (ref: mucinous) & $P=0.103$ & $P=0.363$ \\
\hline PNI & $\mathrm{P}=0.006, \mathrm{HR} 2.82,95 \% \mathrm{Cl}: 1.35-5.88$ & $P=0.373$ \\
\hline Lack of adjuvant chemotherapy & $\mathrm{P}=0.001, \mathrm{HR} 2.72,95 \% \mathrm{Cl}: 1.51-4.90$ & $\mathrm{P}<0.0001, \mathrm{HR} 6.45,95 \% \mathrm{Cl}: 2.59-16.08$ \\
\hline
\end{tabular}

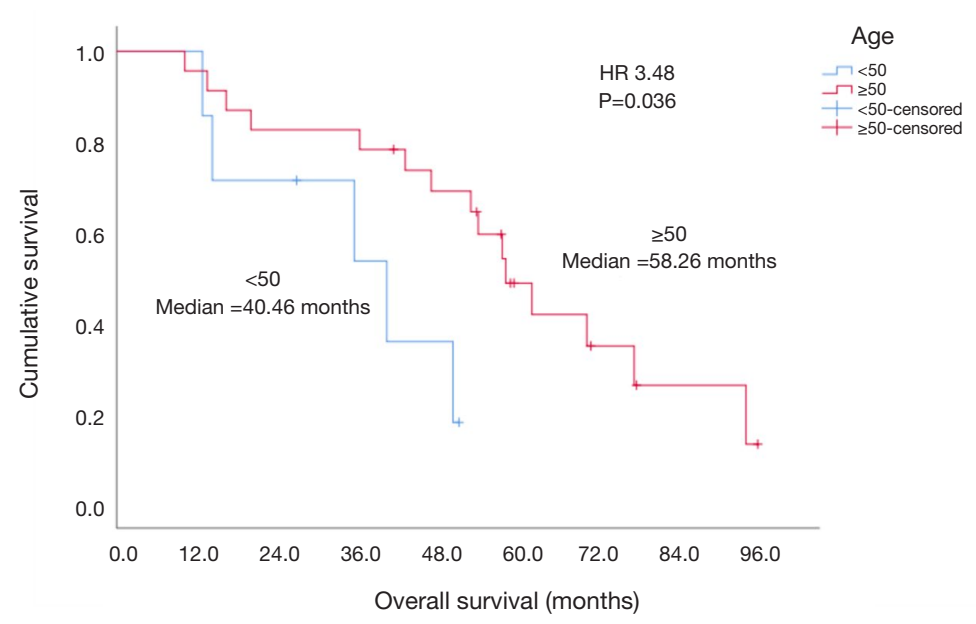

Figure 3 OS for relapsed disease for yRC compared with the standard group. Red: standard group, blue: yRC $(\mathrm{P}=0.036)$.

Table 4 Prognostic factors for overall survival in relapsed disease identified by the univariate and multivariate analyses

\begin{tabular}{lll}
\hline Variable & Univariate analysis & Multivariate analysis \\
\hline Age (ref: yRC) & $\mathrm{P}=0.036, \mathrm{HR}$ 3.96, 95\% Cl: 1.087-11.151 & $\mathrm{P}=0.030, \mathrm{HR} 3.91,95 \% \mathrm{Cl}: 1.14-13.43$ \\
Gender (ref: male) & $\mathrm{P}=0.098$ & $\mathrm{P}=0.512$ \\
Histopathology (ref: mucinous) & $\mathrm{P}=0.125$ & $\mathrm{P}=0.029, \mathrm{HR} 5.69,95 \% \mathrm{Cl}: 1.20-26.52$ \\
Liver metastases & $\mathrm{P}=0.380$ & $\mathrm{P}=0.763$ \\
Surgical treatment (ref: lack of surgical treatment) & $\mathrm{P}=0.029, \mathrm{HR}$ 4.113, 95\% Cl: 1.174-14.414 & $\mathrm{P}=0.030, \mathrm{HR} 4.58,95 \% \mathrm{Cl}: 1.16-18.09$ \\
\hline
\end{tabular}

Our findings are in keeping with work by Lieu et al. who analysed pooled data from 24 different clinical trials of early CRC and demonstrated poorer RFS for younger patients (26). We also corroborate some of the findings by You et al. who compared outcomes of both locally advanced and stage IV rectal cancer in patients $<50$ years to those older than 65 years, who were treated with multimodality therapy. In their study, yRC also had poorer RFS on univariate analysis (19).

Our large dataset of colonoscopies in young patients confirmed the low overall incidence of sporadic CRC in this age group. Young patients were more likely to present 
with left sided disease (data not shown). An analysis from the Mayo Clinic registry demonstrated a similar tendency for yCRC to develop left sided tumours (17). All of the young patients diagnosed with rectal cancer in our study were symptomatic and a large proportion had per rectal bleeding as their predominant complaint, a finding also supported by others $(27,28)$. Another study by Kaplan et $a l$. also demonstrated similarities in presenting symptoms in adolescents and young adults (AYA) (using a cut-off age of 25 years old) compared with older patients (2). This highlights that young patients do develop CRC without predisposing high risk syndromes and present with similar complaints as older patients.

In our study, significantly more yRC had MSI-high tumours compared with older patients and this may lend an explanation for the poorer outcomes seen in yRC. Lynchlike tumours have a higher preponderance in MSI high rectal primaries (29). Recent retrospective studies have demonstrated that MSI-high patients do not derive benefit from 5-FU based chemotherapy (29-31) possibly due to a lack of DNA mismatch repair enzymes that are required for cell cycle arrest and apoptosis following exposure to 5-FU therapy (32).

Given that we focussed on LARC, our study was not powered to detect significant differences in mucinous and non-mucinous histopathology between the two groups, however, in the relapsed cohort, mucinous histology and yRC were associated with shorter OS on multivariate analysis. Other studies have demonstrated a greater tendency for yCRC to present with mucinous and signet ring adenocarcinomas $(2,33,34)$. These tumours tend to present at later stages, are inherently resistant to standard of care therapies and carry a poorer prognosis (35).

Forecasting by Bailey et al. predict a continued rise in CRC incidence for patients younger than 50. By 2023, a diagnosis of 1 in 4 rectal cancers and 1 in 10 colon cancers in this age group is likely to be made $(36,37)$. Whilst microsatellite instability and mucinous pathology lend an explanation for the poorer outcomes seen in this group, they may serve only as surrogate markers. Future retrospective and prospective translational and clinical research in this field should explore biological reasons for the disparities in outcomes in this age group $(26,33,38)$.

Early relapse and poorer PFS secondary to the development of distant metastases were seen in $\mathrm{yRC}$ in our study, demonstrating poor response to standard of care therapies. To improve RFS in LARC, recent clinical trials have assessed the use of neoadjuvant systemic chemotherapy with FOLFOX monotherapy or in combination with radiotherapy. Unfortunately, despite an improvement in local disease control, these trials did not translate into improved RFS $(39,40)$. There is preclinical evidence to support the beneficial role of checkpoint inhibitors either administered concomitantly with, or at the end of, radiotherapy in mouse models of colorectal carcinoma (41). This has been taken to clinical trials exploring the addition of immunotherapy to determine whether it augments clinical response to standard pre-operative therapy (ClinicalTrials.gov registry: NCT04293419, NCT03299660, NCT03854799).

The advantage of our population-based study is that it did not rely upon a database for information, rather, detailed data about treatment, morbidity, performance status and other information was obtained. We reviewed data from multiple sites, including two of the largest tertiary referral centres in Sydney, as well as from a larger dataset of colonoscopies, which enabled us to capture all colonoscopies performed and rectal cancers detected. Further, we focussed on LARC as a specific subgroup, the risk of which is rising significantly in incidence in yCRC and remains an area of unmet need.

Due to the study design several limitations exist. Given the retrospective nature of this study, unmeasured confounders may exist, which were not accounted for in this study design. Further, given the small sample size, there may be limitations in detecting small differences in survival between the two cohorts.

\section{Conclusions}

Our study provides preliminary data to suggest that young patients with LARC have poorer treatment outcomes compared with older patients. Patients under 50 years with LARC are more likely to have MSI tumours with a more aggressive disease course and in our study demonstrated poorer RFS. Further, yRC with relapsed disease demonstrated poorer PFS and OS. Initiatives to improve early detection of these patients may improve outcomes.

Given the rising incidence of left sided yCRC, which is dominated by rectal primaries, our study highlights the need for further research to understand the biology of the disease and correlate that with measurable outcomes and to identify novel neoadjuvant and adjuvant treatments for locally advanced yRC. 


\section{Acknowledgments}

Funding: None.

\section{Footnote}

Reporting Checklist: The authors have completed the STROBE reporting checklist. Available at http://dx.doi. org/10.21037/jgo-20-300

Data Sharing Statement: Available at http://dx.doi. org/10.21037/jgo-20-300

Conflicts of Interest: All authors have completed the ICMJE uniform disclosure form (available at http://dx.doi. org/10.21037/jgo-20-300). The authors have no conflicts of interest to declare.

Ethical Statement: The authors are accountable for all aspects of the work in ensuring that questions related to the accuracy or integrity of any part of the work are appropriately investigated and resolved. The study was conducted in accordance with the Declaration of Helsinki (as revised in 2013). The study was approved by the institutional ethics board of the Western Sydney Local Health District (IBR: 1811-15 QA). No informed consent was required as this was a retrospective audit.

Open Access Statement: This is an Open Access article distributed in accordance with the Creative Commons Attribution-NonCommercial-NoDerivs 4.0 International License (CC BY-NC-ND 4.0), which permits the noncommercial replication and distribution of the article with the strict proviso that no changes or edits are made and the original work is properly cited (including links to both the formal publication through the relevant DOI and the license). See: https://creativecommons.org/licenses/by-nc-nd/4.0/.

\section{References}

1. Feletto E, Yu XQ, Lew JB, et al. Trends in Colon and Rectal Cancer Incidence in Australia from 1982 to 2014: Analysis of Data on Over 375,000 Cases. Cancer Epidemiol Biomarkers Prev 2019;28:83-90.

2. Kaplan MA, Isikdogan A, Gumus M, et al. Childhood, adolescents, and young adults $(</=25 \mathrm{y})$ colorectal cancer: study of Anatolian Society of Medical Oncology. J Pediatr Hematol Oncol 2013;35:83-9.

3. Kuipers EJ, Grady WM, Lieberman D, et al. Colorectal cancer. Nature reviews Disease primers 2015;1:15065.

4. WHO. Cancer. 2018. Available online: https://www.who. int/health-topics/cancer

5. Siegel RL, Torre LA, Soerjomataram I, et al. Global patterns and trends in colorectal cancer incidence in young adults. Gut 2019;68:2179-85.

6. Guinney J, Dienstmann R, Wang X, et al. The consensus molecular subtypes of colorectal cancer. Nat Med 2015;21:1350-6.

7. Dienstmann R, Vermeulen L, Guinney J, et al. Consensus molecular subtypes and the evolution of precision medicine in colorectal cancer. Nat Rev Cancer 2017;17:79-92.

8. Cancer Genome Atlas N. Comprehensive molecular characterization of human colon and rectal cancer. Nature 2012;487:330-7.

9. Sato JD, Kawamoto T, Le AD, et al. Biological effects in vitro of monoclonal antibodies to human epidermal growth factor receptors. Mol Biol Med 1983;1:511-29.

10. Kapiteijn E, Marijnen CA, Nagtegaal ID, et al. Preoperative radiotherapy combined with total mesorectal excision for resectable rectal cancer. $\mathrm{N}$ Engl J Med 2001;345:638-46.

11. Presta LG, Chen H, O'Connor SJ, et al. Humanization of an anti-vascular endothelial growth factor monoclonal antibody for the therapy of solid tumors and other disorders. Cancer Res 1997;57:4593-9.

12. Endreseth BH, Romundstad P, Myrvold HE, et al. Rectal cancer in the young patient. Dis Colon Rectum 2006;49:993-1001.

13. Lee $\mathrm{KC}, \mathrm{Ou} \mathrm{YC}, \mathrm{Hu} \mathrm{WH}$, et al. Meta-analysis of outcomes of patients with stage IV colorectal cancer managed with chemotherapy/radiochemotherapy with and without primary tumor resection. Onco Targets Ther 2016;9:7059-69.

14. Siegel RL, Fedewa SA, Anderson WF, et al. Colorectal Cancer Incidence Patterns in the United States, 19742013. J Natl Cancer Inst 2017;109:djw322.

15. Barreto SG. Young-onset rectal cancer patients: in need of answers. Future Oncol 2019;15:1053-5.

16. Murphy CC, Wallace K, Sandler RS, et al. Racial Disparities in Incidence of Young-Onset Colorectal Cancer and Patient Survival. Gastroenterology 2019;156:958-65.

17. Kasi PM, Shahjehan F, Cochuyt JJ, et al. Rising Proportion of Young Individuals With Rectal and Colon Cancer. Clin Colorectal Cancer 2019;18:e87-95.

18. O'Connell JB, Maggard MA, Liu JH, et al. Are survival rates different for young and older patients with rectal cancer? Dis Colon Rectum 2004;47:2064-9. 
19. You YN, Dozois EJ, Boardman LA, et al. Young-onset rectal cancer: presentation, pattern of care and long-term oncologic outcomes compared to a matched older-onset cohort. Ann Surg Oncol 2011;18:2469-76.

20. Al-Barrak J, Gill S. Presentation and outcomes of patients aged 30 years and younger with colorectal cancer: a 20year retrospective review. Med Oncol 2011;28:1058-61.

21. Yeo SA, Chew MH, Koh PK, et al. Young colorectal carcinoma patients do not have a poorer prognosis: a comparative review of 2,426 cases. Tech Coloproctol 2013;17:653-61.

22. Chung YF, Eu KW, Machin D, et al. Young age is not a poor prognostic marker in colorectal cancer. Br J Surg 1998;85:1255-9.

23. Fu J, Yang J, Tan Y, et al. Young patients ( $\leq 35$ years old) with colorectal cancer have worse outcomes due to more advanced disease: a 30-year retrospective review. Medicine 2014;93:e135.

24. Bleyer A, Ferrari A, Whelan J, et al. Global assessment of cancer incidence and survival in adolescents and young adults. Pediatr Blood Cancer 2017;64:e26497.

25. Xynos E, Tekkis P, Gouvas N, et al. Clinical practice guidelines for the surgical treatment of rectal cancer: a consensus statement of the Hellenic Society of Medical Oncologists (HeSMO). Ann Gastroenterol 2016;29:103-26.

26. Lieu CH, Renfro LA, de Gramont A, et al. Association of Age With Survival in Patients With Metastatic Colorectal Cancer: Analysis From the ARCAD Clinical Trials Program. J Clin Oncol 2014;32:2975-84.

27. Chen FW, Sundaram V, Chew TA, et al. Advanced-Stage Colorectal Cancer in Persons Younger Than 50 Years Not Associated With Longer Duration of Symptoms or Time to Diagnosis. Clin Gastroenterol Hepatol 2017;15:728-37.e3.

28. Dozois EJ, Boardman LA, Suwanthanma W, et al. Youngonset colorectal cancer in patients with no known genetic predisposition: can we increase early recognition and improve outcome? Medicine 2008;87:259-63.

29. Samowitz WS, Curtin K, Wolff RK, et al. Microsatellite instability and survival in rectal cancer. Cancer Causes Control 2009;20:1763-8.

30. Ribic CM, Sargent DJ, Moore MJ, et al. Tumor microsatellite-instability status as a predictor of benefit from fluorouracil-based adjuvant chemotherapy for colon cancer. N Engl J Med 2003;349:247-57.

31. de Vos tot Nederveen Cappel WH, Meulenbeld HJ, Kleibeuker JH, et al. Survival after adjuvant 5-FU treatment for stage III colon cancer in hereditary nonpolyposis colorectal cancer. Int J Cancer 2004;109:468-71.

32. Carethers JM, Smith EJ, Behling CA, et al. Use of 5-fluorouracil and survival in patients with microsatelliteunstable colorectal cancer. Gastroenterology 2004;126:394-401.

33. Chan KK, Dassanayake B, Deen R, et al. Young patients with colorectal cancer have poor survival in the first twenty months after operation and predictable survival in the medium and long-term: analysis of survival and prognostic markers. World J Surg Oncol 2010;8:82.

34. Wang R, Wang MJ, Ping J. Clinicopathological Features and Survival Outcomes of Colorectal Cancer in Young Versus Elderly: A Population-Based Cohort Study of SEER 9 Registries Data (1988-2011). Medicine (Baltimore) 2015;94:e1402.

35. Nitsche U, Zimmermann A, Spath C, et al. Mucinous and signet-ring cell colorectal cancers differ from classical adenocarcinomas in tumor biology and prognosis. Ann Surg 2013;258:775-82; discussion 782-3.

36. Bailey $\mathrm{CE}, \mathrm{Hu} \mathrm{CY}$, You $\mathrm{YN}$, et al. Increasing disparities in the age-related incidences of colon and rectal cancers in the United States, 1975-2010. JAMA Surg 2015;150:17-22.

37. Inra JA, Syngal S. Colorectal cancer in young adults. Dig Dis Sci 2015;60:722-33.

38. You YN, Xing Y, Feig BW, et al. Young-onset colorectal cancer: is it time to pay attention? Arch Intern Med 2012;172:287-9.

39. Franke AJ, Parekh H, Starr JS, et al. Total Neoadjuvant Therapy: A Shifting Paradigm in Locally Advanced Rectal Cancer Management. Clin Colorectal Cancer 2018;17:1-12.

40. Deng Y, Chi P, Lan P, et al. Modified FOLFOX6 with or without radiation in neoadjuvant treatment of locally advanced rectal cancer: Final results of the Chinese FOWARC multicenter randomized trial. J Clin Oncol 2018;36:3502.

41. Dovedi SJ, Adlard AL, Lipowska-Bhalla G, et al. Acquired resistance to fractionated radiotherapy can be overcome by concurrent PD-L1 blockade. Cancer Res 2014;74:5458-68.

Cite this article as: Habib R, Burgess NG, Bourke MJ, Wong M, Wilcken N, Toh J, El-Khoury T, Pathma-Nathan N, Ctercteko G, Jayamohan J, Micklethwaite K, Nagrial A. Outcomes of young patients diagnosed with locally advanced rectal cancer. J Gastrointest Oncol 2021;12(2):592-601. doi: 10.21037/jgo-20-300 
Supplementary

Table S1 Postoperative clinicopathologic characteristics for $\mathrm{yRC}(\mathrm{n}=25)$ compared with standard group $(\mathrm{n}=74)$

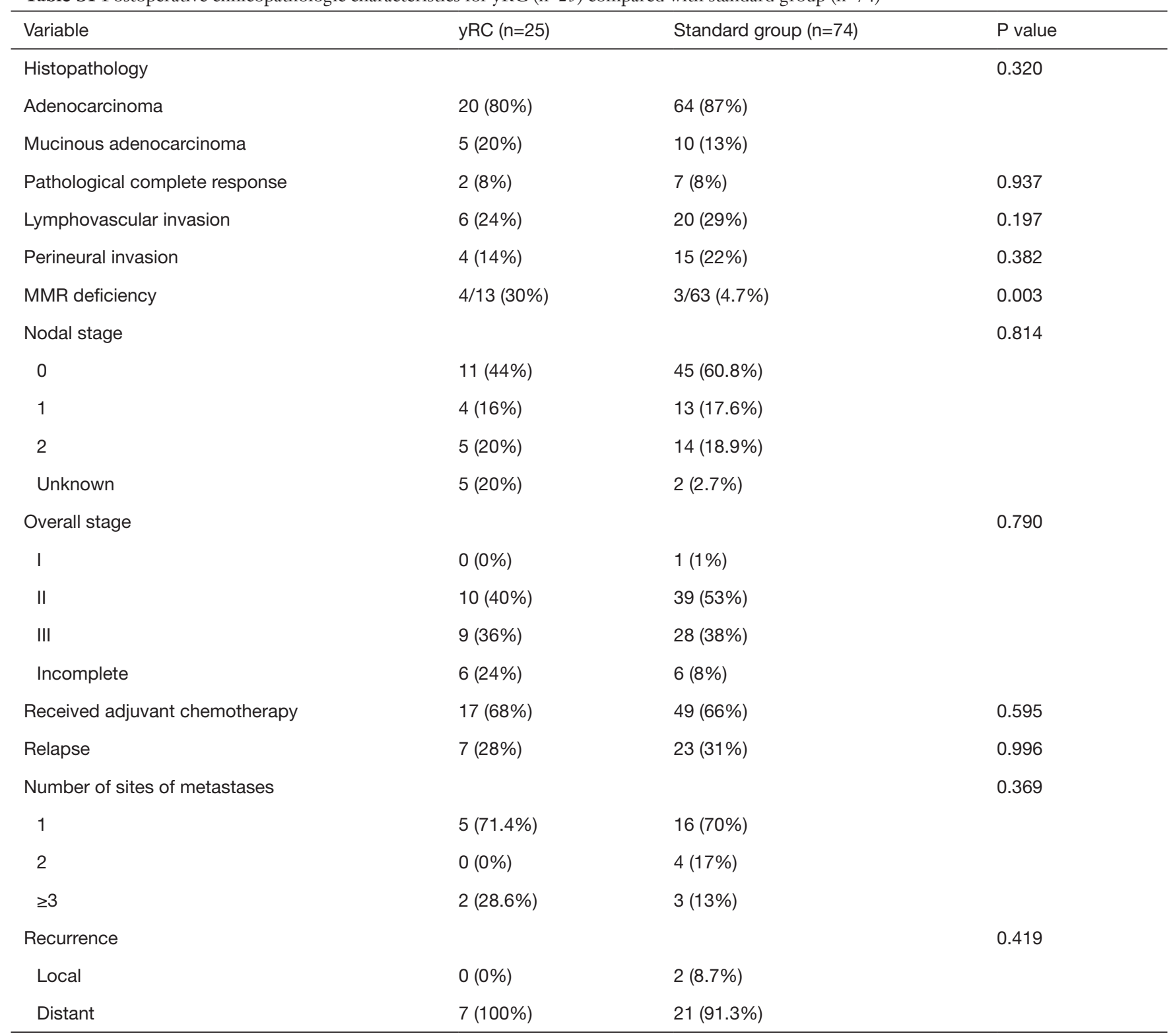

